\title{
Vlasov simulation of kinetic shear Alfvén waves
}

\author{
Tilman Dannert and Frank Jenko \\ Max-Planck Institut für Plasmaphysik, EURATOM association, \\ D-85748 Garching
}

\begin{abstract}
The treatment of kinetic shear Alfvén waves in homogeneous magnetized plasmas by means of Vlasov simulation is examined. To this end, the driftkinetic version of the Vlasov-Maxwell equations is solved via various numerical schemes, all employing a grid in (1+1)D phase space. Since kinetic shear Alfvén waves are Landau damped, the use of an equidistant grid in velocity space leads to a recurrence problem. The latter can be circumvented, however, by damping the finest velocity space scales through higher-order collision operators. Of particular interest is the question if and under which circumstances the magnetohydrodynamic limit (small perpendicular wavenumber) can be recovered.
\end{abstract}

Key words: 52.35.-g, 52.65.Tt, Alfvén waves, Vlasov simulation

\section{Introduction}

Magnetized plasmas exhibit a large number of waves and instabilities.[1] In the low beta, low frequency range, $\beta \ll 1$ and $\omega \ll \Omega_{i}$ [where $\Omega_{i}=\left(q_{i} B\right) /\left(m_{i} c\right)$ is the ion cyclotron frequency], shear Alfvén waves are of particular interest since they are involved in a large number of plasma physics problems. [2] While their simplest description is given by magnetohydrodynamics (MHD), kinetic effects come into play once their perpendicular wavelength reaches the drift wave dispersion scale $\rho_{s}=c_{s} / \Omega_{i}$ [where $c_{s}=\left(T_{e} / m_{i}\right)^{1 / 2}$ is the ion sound speed in the cold ion limit]. In this regime, they are therefore called kinetic shear Alfvén waves.[2] If the perpendicular wavelength is further descreased, they finally transition into electron sound waves. These are exact analogues of ion sound waves, with the role of electrons and ions reversed. Both kinetic shear Alfvén waves and electron sound waves are Landau damped. 
Kinetic shear Alfvén waves are described by the driftkinetic (or gyrokinetic) version of the Vlasov-Maxwell equations in $(3+2) D$ phase space (see, e.g., Refs. $[3,4])$. In both cases, the fast gyrophase dependence is removed analytically. For the purposes of this paper, it will suffice to study a homogeneous magnetized plasma whose $(1+1) \mathrm{D}$ phase space is spanned by the fieldline-following coordinate $z$ and the parallel velocity $v_{\|}$. The perpendicular wavenumber enters only as a parameter, and the remaining velocity space variable can be integrated out.[5,6] Despite its relative simplicity, this reduced system still contains the key challenges one has to face if seeking a numerical representation of kinetic shear Alfvén waves.

Several options are available when trying to solve the corresponding initial value problem by means of computer simulations. Probably the most common approach is the particle-in-cell (PIC) method.[7] As it turns out, however, the inherent particle noise prevents a straightforward solution unless the number of particles is increased substantially. As an alternative route, various noisereduced PIC schemes have been proposed (see Ref. [8] and references therein), but the basic problem remains. Vlasov methods, on the other hand, avoid the noise issue completely by employing a fixed grid in phase space.[9] The basic integro-differential equations are then finite differenced and solved via techniques borrowed from computational fluid dynamics. Here, we adopt this latter approach.

The paper is structured as follows. In Section 2, the basic equations are introduced and the dispersion relation for kinetic shear Alfvén waves in a homogeneous magnetized plasma is derived. In Section 3, different phase space discretization schemes are investigated, involving explicit Runge-Kutta time stepping and upwind or central spatial discretizations. Due to the use of an equidistant grid in velocity space, a recurrence problem is encountered which can be circumvented, however, by the use of appropriate hypercollision operators. In Section 4, convergence with respect to resolution in velocity space and real space is checked. It turns out that the number of required velocity space points is surprisingly low. The results of the initial value computations are compared to those of the repective dispersion relation for a wide range of physical parameters in Section 4. Finally, a brief summary is presented in Section 6 . 


\section{Basic equations}

\subsection{Unnormalized equations in $(3+2) D$ phase space}

Kinetic shear Alfvén waves in a homogeneous magnetoplasma are described by the driftkinetic version of the Vlasov-Maxwell equations in $(3+2) \mathrm{D}$ phase space. The latter is spanned by the three spatial variables $(x, y, z)$ [the $z$-axis is assumed to be aligned with the background magnetic field] and two velocity space variables, e.g. $\left(v_{\|}, v_{\perp}\right)$. In the low-frequency limit, the fast gyrophase dependence may be removed analytically. Moreover, for simplicity, the ions are taken to be singly charged and immobile. After linearization, one is then left with the driftkinetic Vlasov equation

$$
\frac{\partial F_{e}}{\partial t}+v_{\|} \frac{\partial F_{e}}{\partial z}-\frac{e E_{\|}}{m_{e}} \frac{\partial F_{e 0}}{\partial v_{\|}}=0, \quad E_{\|}=-\frac{\partial \phi}{\partial z}-\frac{1}{c} \frac{\partial A_{\|}}{\partial t}
$$

for the perturbed electron distribution function $F_{e}$, together with the corresponding field equations

$$
\frac{n_{e 0} m_{i} c^{2}}{e B^{2}} \nabla_{\perp}^{2} \Phi=\int F_{e} d v_{\|}, \quad \nabla_{\perp}^{2} A_{\|}=\frac{4 \pi e}{c} \int v_{\|} F_{e} d v_{\|}
$$

for the perturbed electrostatic and parallel magnetic potentials, $\phi$ and $A_{\|}$. Here, $e>0$ is the elementary charge and $F_{e 0}$ is the equilibrium distribution function of the electrons which is assumed to be given by an unshifted Maxwellian,

$$
F_{e 0}=n_{e 0}\left(\pi v_{T e}^{2}\right)^{-3 / 2} e^{-v^{2} / v_{T e}^{2}},
$$

such that $\partial_{v_{\|}} F_{e 0}=-m_{e} v_{\|} F_{e 0} / T_{e 0}$. Here, $v_{T e}^{2}=2 T_{e 0} / m_{e}$ and $v^{2}=v_{\|}^{2}+v_{\perp}^{2}$.

\subsection{Normalization and reduction to $(1+1) D$ phase space}

Table 1

\begin{tabular}{|ccccccc|}
\hline$x, y$ & $z$ & $v_{\|}, v_{\perp}$ & $t$ & $g_{e}, F_{e}, F_{e 0}$ & $\phi$ & $A_{\|}$ \\
\hline$\rho_{s}$ & $L_{\|}$ & $v_{T e}$ & $L_{\perp} / c_{s}$ & $n_{e 0} / v_{T e}^{3}$ & $T_{e 0} / e$ & $\rho_{s} B_{0} \beta_{e} L_{\|} / L_{\perp}$ \\
\hline
\end{tabular}

Normalization of all independent and dependent variables (cp. Refs. $[5,6]$ ).

For reasons that will become clear in Section 3, we introduce the modified distribution function $g_{e}=F_{e}-\left(v_{\|} / c\right)\left(e A_{\|} / T_{e 0}\right) F_{e 0} \cdot[6,10]$ Normalizing Eqs. (1$3)$ according to Table 1 , one thus obtains

$$
\frac{\partial g_{e}}{\partial t}+\alpha_{e} v_{\|} \nabla_{\|}\left(F_{e}-\phi F_{e 0}\right)=0, \quad g_{e}=F_{e}-\alpha_{e} v_{\|} \hat{\epsilon} \beta_{e} A_{\|} F_{e 0}
$$


together with

$$
\nabla_{\perp}^{2} \phi=\int g_{e} d v_{\|}, \quad\left(\nabla_{\perp}^{2}-\beta_{e} / \mu_{e}\right) A_{\|}=\int \alpha_{e} v_{\|} g_{e} d v_{\|}
$$

Here, we have used the following definitions:

$$
\beta_{e}=\frac{4 \pi n_{e 0} T_{e 0}}{B_{0}^{2}}, \quad \mu_{e}=\frac{m_{e}}{m_{i}}, \quad \hat{\epsilon}=\left(\frac{L_{\|}}{L_{\perp}}\right)^{2}, \quad \alpha_{e}^{2}=\frac{2}{\hat{\epsilon} \mu_{e}} .
$$

As mentioned before, $c_{s}^{2}=T_{e 0} / m_{i}, \rho_{s}=c_{s} / \Omega_{i}$, and $\Omega_{i}=\left(e B_{0}\right) /\left(m_{i} c\right)$. The normalizations shown in Table 1 correspond to the ones used in the nonlinear gyrokinetic code gene (see also Refs. $[5,6]$ ). Note that due to the use of $g_{e}$ instead of $F_{e}$ in Eq. (5), the $\beta_{e} / \mu_{e}$ term appears in Ampére's law.

Finally, we further simplify the problem by Fourier transforming the $x$ and $y$ directions, and by integrating out $v_{\perp}$ space. This procedure turns Eq. (5) into

$$
k_{\perp}^{2} \phi=-\int g_{e} d v_{\|}, \quad\left(k_{\perp}^{2}+\beta_{e} / \mu_{e}\right) A_{\|}=-\int \alpha_{e} v_{\|} g_{e} d v_{\|}
$$

Eq. (4) is now to be interpreted as an equation for $g_{e}\left(z, v_{\|}, t\right)$, and $F_{e 0}=$ $\pi^{-1 / 2} e^{-v_{\|}^{2}}$. Although the $z$ direction could also be Fourier transformed, we refrain from doing so because the resulting $(0+1) \mathrm{D}$ problem turns out to possess different numerical properties. In particular, one finds that schemes which work for the $(0+1) D$ problem may exhibit numerical instabilities when applied to the $(1+1) \mathrm{D}$ case. To be able to generalize the approaches discussed below to inhomogeneous situations, it is therefore vital to keep the $z$ coordinate.

\subsection{Dispersion relation and nominal parameters}

Using Eqs. (4) and (6), one may easily derive the dispersion relation of kinetic shear Alfvén waves in a homogeneous magnetoplasma. It reads

$$
k_{\perp}^{2}+[1+\bar{\omega} Z(\bar{\omega})]\left[1-2 \bar{\omega}^{2}\left(\beta_{e} / \mu_{e}\right)\right]=0
$$

where $\bar{\omega}=\omega /\left(\alpha_{e} k_{\|}\right)$and $Z$ is the well-known plasma dispersion function.[11] Obviously, the $(1+1) \mathrm{D}$ system is really characterized by only two parameters, $k_{\perp}$ and $\beta_{e} / \mu_{e}$. Changes in $\alpha_{e}$ and $k_{\|}$may be accounted for by merely renormalizing the complex frequency $\omega$. Throughout this paper, we use $k_{\|}=1$ and $\mu_{e}=1 / 1836$, and with the exception of Section 5.3 we choose $\hat{\epsilon}=10^{4}$ which corresponds to $\alpha_{e} \approx 0.606$. Setting $k_{x}=0, k_{\perp}$ and $k_{y}$ will be used synonymously. 


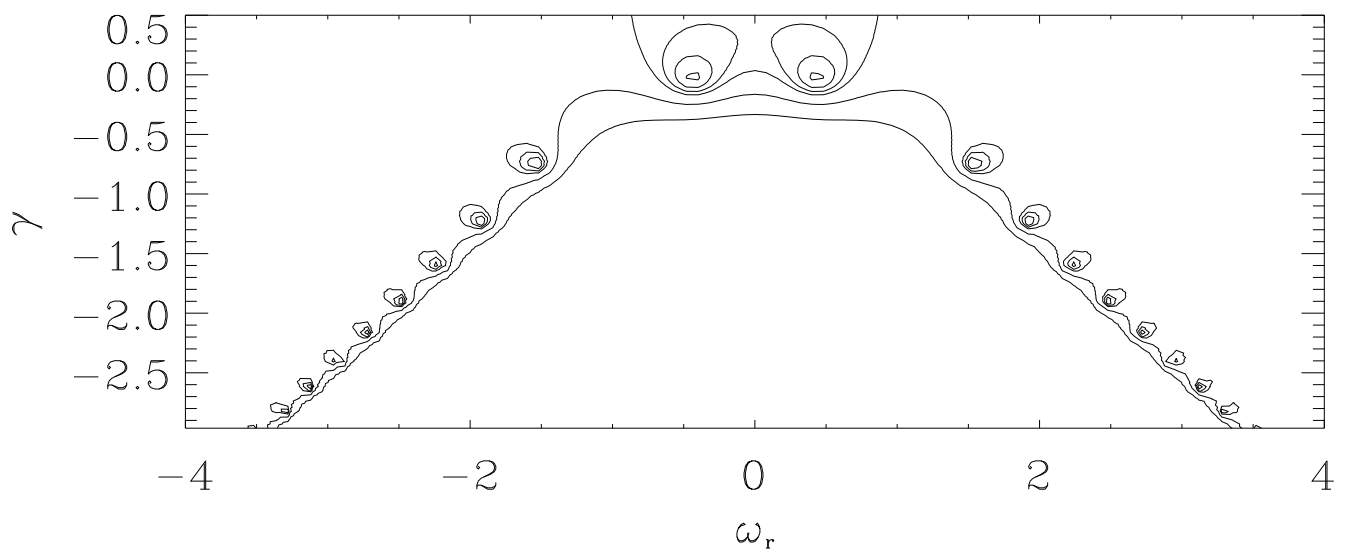

Fig. 1. Contour plot of the absolute value of the left-hand side of Eq. (7). The zeros of this function correspond to solutions of the dispersion relation.

Given $k_{\perp}$ and $\beta_{e} / \mu_{e}$, the task is to determine all positions in the complex $\bar{\omega}$ plane at which both the real part and the imaginary part of Eq. (7) vanish simultaneously. In our case, this is done by means of Newton's method. A typical contour plot of the absolute value of the left-hand side of Eq. (7) is shown in Fig. 1. Here, $k_{\perp}=0.3$ and $\beta_{e} / \mu_{e}=1$. Note that the $Z$ function allows for an infinite number of solutions. However, in the present context we are only interested in the least damped modes (i.e., the ones with the largest imaginary part) which correspond to the kinetic shear Alfvén waves. In the remainder of this paper, such solutions will be used to assess the results of initial value computations.

\section{Numerical solution of the initial value problem}

\subsection{Parallel canonical momentum method}

The numerical solution of Eqs. (4) and (6) as an initial value problem is not straightforward. In particular, the partial time derivative of $A_{\|}$in Eq. (4) must be treated with care if a time explicit scheme is to be used. As has been known since the 1970's, a simple extrapolation from past values of $A_{\|}$ leads to numerical schemes which are violently unstable.[12] This problem may be circumvented, however, by employing the parallel canonical momentum method proposed in Refs. [6,10]. Combining $\partial_{t} F_{e}$ and $\partial_{t} A_{\|}$, Eq. (4) may be used to step the modified distribution function $g_{e}$. Next, the potentials $\phi$ and $A_{\|}$at the next time level may be computed from Eq. (6). Knowing the updated values of both $g_{e}$ and $A_{\|}$, the new $F_{e}$ may finally be obtained. All numerical schemes used in this paper are based on this idea. 
Like some alternative approaches (see Ref. [10] and references therein), the parallel canonical momentum method involves an important subtlety which becomes important at large $\beta_{e} / \mu_{e}$ and/or at small $k_{\perp}$. When $F_{e}$ was replaced by $g_{e}$ in Ampère's law, the $\beta_{e} / \mu_{e}$ term was introduced analytically to cancel the $A_{\|}$contribution to the first moment of $g_{e}$. However, if the second (numerically computed) moment of $F_{e 0}$ deviates from $1 / 2$, wrong answers for $\omega$ are obtained in the high $\beta$, long wavelength limit. Therefore, one has to make sure that such residuals are avoided, either by using very accurate integrators or by replacing the analytical value for $\int v_{\|}^{2} \exp \left(-v_{\|}^{2}\right) d v_{\|}$in Eq. (6) by its numerical counterpart.

In the present work, we make use of this latter idea, computing the $v_{\|}$integrals in Eq. (6) via a simple trapezoidal rule. The $z$ domain is taken to be periodic and runs from $-\pi$ to $\pi$, while $\left|v_{\|}\right| \leq v_{\text {cut }}$. Unless noted otherwise, we set $v_{\text {cut }}=3$. Equidistant grids are used in all three dimensions, and the grid spacings corresponding to $t, v_{\|}$, and $z$ are called, respectively, $\Delta t, \Delta v$, and $h$. So e.g. the value of $g_{e}$ at the time level $t_{n}$ and at the phase space point $\left(v_{i}, z_{m}\right)$ is denoted by $g_{i, m}^{(n)}$ where $v_{i}=\left(2 i-N_{v}-1\right) \Delta v / 2\left[i=1, \ldots, N_{v}\right]$ and $z_{m}=\left(2 m-N_{z}-1\right) h / 2\left[m=1, \ldots, N_{z}\right]$. While many time implicit methods are also available, we will restrict to time explicit schemes here since they allow for straightforward generalizations to more complex problems. A very flexible approach in which the discretizations in phase space and time are decoupled is the 'method of lines.'

\subsection{Method of lines}

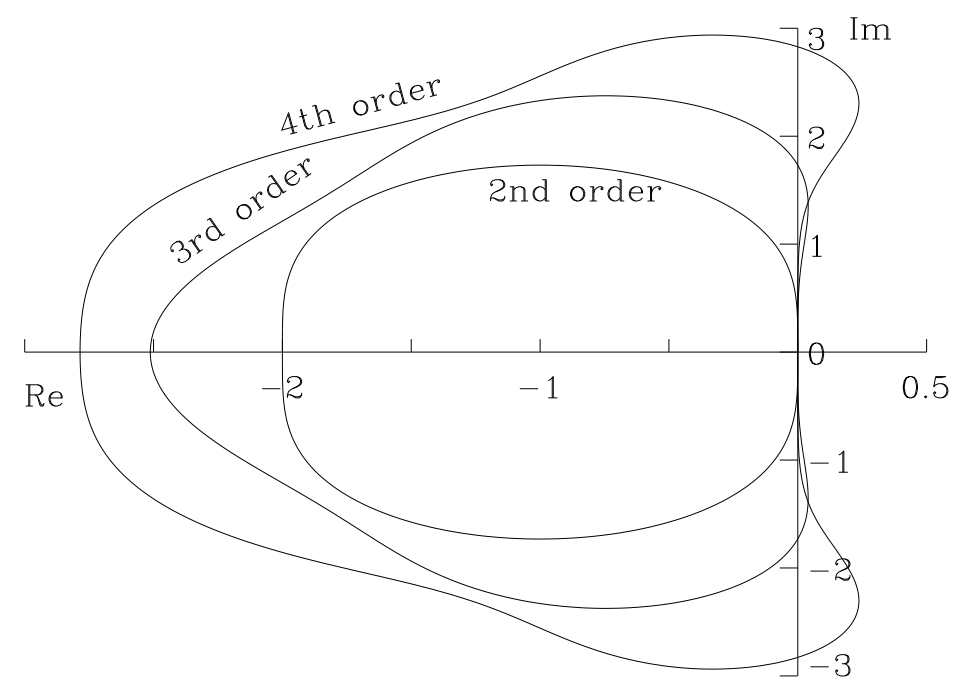

Fig. 2. Low-order explicit Runge-Kutta methods: Stability regions in the complex plane. 
Eq. (4) may be rewritten as

$$
\frac{\partial g}{\partial t}=-\alpha_{e} v_{\|} \nabla_{\|}\left(g+\alpha_{e} v_{\|} \hat{\epsilon} \beta_{e} A_{\|} F_{0}-\phi F_{0}\right)
$$

where the species subscript 'e' has been omitted. Together with Eq. (6), it constitutes an integro-differential equation for $g$. Introducing a homogeneous grid in phase space (like outlined above) but keeping time as a continuous variable, Eq. (8) turns into a linear system of ordinary differential equations,

$$
\frac{\partial \mathbf{g}}{\partial t}=M \mathbf{g}
$$

Here, $\mathbf{g}$ is a $N_{z} N_{v}$-dimensional vector and $M$ is a $N_{z} N_{v} \times N_{z} N_{v}$ matrix. The latter consists of $N_{z} \times N_{z}$ submatrices whose structure depends on the discretization of the spatial differential operator $\nabla_{\|}$as it is applied to $g, A_{\|}$, and $\phi$. To solve Eq. (9), one may choose from a wide variety of well-documented time advance methods. In the following, we will focus on explicit Runge-Kutta (ERK) methods [13] which are known to be fairly robust and rather straightforward to implement. Moreover, they allow for easy time step adjustments which are sometimes necessary in nonlinear generalizations of the computations presented here.[14] ERK schemes are known to be linearly stable if and only if all eigenvalues of the matrix $M$ (multiplied by the time step $\Delta t$ ) fall inside a certain stability region in the complex plane. The stability regions of low-order ERK methods are shown in Fig. 2 (see Ref. [13]). As will be shown below, centered finite-difference representations of $\nabla_{\|}$lead to purely imaginary eigenvalues. This means that the employed ERK scheme needs to be at least of third order to be able to handle such cases. The time advance method used throughout this paper is the so-called Heun scheme, a classic representative of the one-parameter family of third-order ERK schemes. In the language of Eq. (9), it reads:

$$
\begin{aligned}
\mathbf{k}_{1} & =M \mathbf{g}^{(n)} \\
\mathbf{k}_{2} & =M\left(\mathbf{g}^{(n)}+\frac{\Delta t}{3} \mathbf{k}_{1}\right) \\
\mathbf{k}_{3} & =M\left(\mathbf{g}^{(n)}+\frac{2 \Delta t}{3} \mathbf{k}_{2}\right) \\
\mathbf{g}^{(n+1)} & =\mathbf{g}^{(n)}+\frac{\Delta t}{4}\left(\mathbf{k}_{1}+3 \mathbf{k}_{3}\right)
\end{aligned}
$$

So far, we have not addressed the question which finite difference schemes are to be used to represent the action of the spatial differential operator $\nabla_{\|}$on $g$, $A_{\|}$, and $\phi$. As it turns out, one must approach this topic carefully. 


\subsection{Finite difference schemes for $\nabla_{\|}$}

Straightforward finite difference representations of $\nabla_{\|}$are the centered secondorder method given by

$$
\frac{\partial g_{l, m}}{\partial z}=\frac{g_{l, m+1}-g_{l, m-1}}{2 h}
$$

and the respective fourth-order scheme,

$$
\frac{\partial g_{l, m}}{\partial z}=\frac{g_{l, m-2}-8 g_{l, m-1}+8 g_{l, m+1}-g_{l, m+2}}{12 h} .
$$

Applying Eq. (10) to all terms in Eq. (8), the eigenvalues of the matrix $M$ are found to be purely imaginary as can be seen in Fig. 3(a). This is a reflection of the fact that centered schemes do not involve numerical dissipation. As a consequence, if ERK time advance methods are used, they must be of order three or higher. On the other hand, (computationally less expensive) lowerorder ERK schemes may be used if all eigenvalues exhibit negative real parts. This implies that the finite difference scheme has to have numerical dissipation and leads us to consider asymmetric (or 'upwind') methods.
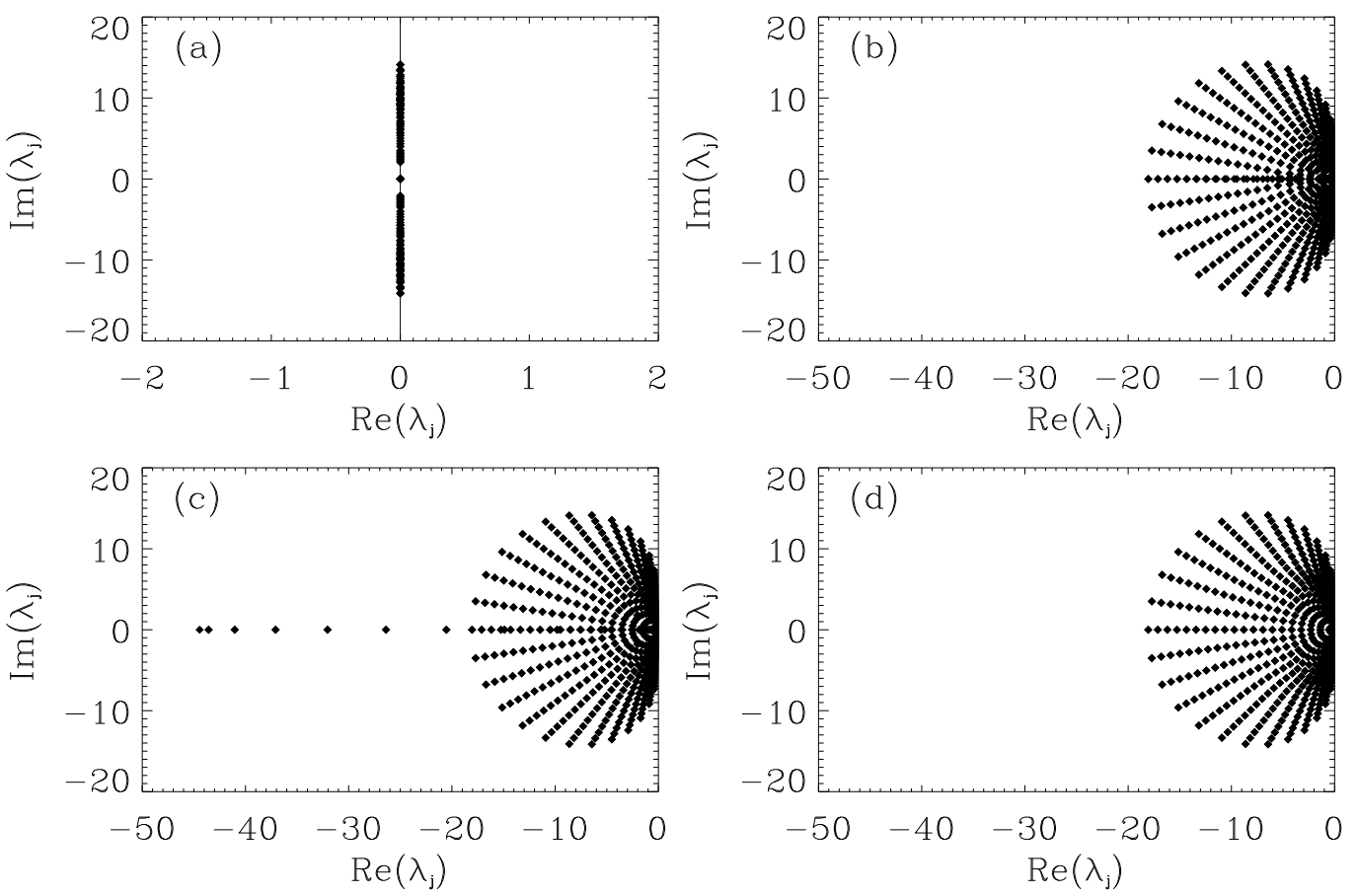

Fig. 3. Numerically computed eigenvalues $\lambda_{j}$ of the phase space matrix $M$ for $k_{\perp}=0.3, \beta_{e} / \mu_{e}=1, N_{z}=32$, and $N_{v}=40$. Here, the second-order upwind scheme of Eq. (12) was used for (a) no term at all, (b) $g$ and $A_{\|}$, (c) $g, A_{\|}$, and $\phi$, (d) $g$ only; the remaining terms have been treated according to Eq. (10).

Upwind methods are often used for advection equations. Here, the information 
reaching a fixed point in space comes from the upwind direction, and the choice of asymmetric weights and/or stencils is supposed to reflect that. A secondorder upwind scheme for $v_{l}>0$ is given by

$$
\frac{\partial g_{l}}{\partial z}=\frac{g_{l, m-2}-5 g_{l, m-1}+3 g_{l, m}+g_{l, m+1}}{4 h}
$$

where $m$ is the space index and $l$ the velocity index. If the same scheme is used for $A_{\|}$, and $\phi$ is center-differenced according to Eq. (10), one arrives at the eigenvalue distribution shown in 3(b). Using an appropriate time step, the rescaled eigenvalues will fit into any of the four stability regions shown in Fig. 2. However, if all terms in Eq. (8) are upwinded, some eigenvalues exhibit large negative real parts, as can be seen in Fig. 3(c). Their magnitude is observed to scale like $k_{\perp}^{-2}$. A third possibility is to upwind only $g$ and to center-difference both potentials. This leads to the eigenvalue distribution shown in Fig. 3(d) which resembles that of case (b).

These findings may be understood by inspecting the matrix $M$ of Eq. (9). If only velocity space is discretized, one obtains

$$
\frac{\partial g_{l}}{\partial t}=-\sum_{i=1}^{N_{v}} \alpha_{e} v_{l}\left[\delta_{l i}+F_{0 l} \Delta v\left(\frac{1}{k_{\perp}^{2}}-\frac{2 v_{l} v_{i}\left(\beta_{e} / \mu_{e}\right)}{k_{\perp}^{2}+\beta_{e} / \mu_{e}}\right)\right] \frac{\partial g_{i}}{\partial z} \equiv-\sum_{i=1}^{N_{v}} V_{l i} \frac{\partial g_{i}}{\partial z}
$$

which can be interpreted as a multidimensional advection equation. Also discretizing the system spatially, an upwind discretization of all terms yields

$$
V_{l l}=\alpha_{e} v_{l}\left[1+F_{0 l} \Delta v\left(\frac{1}{k_{\perp}^{2}}-\frac{2 v_{l}^{2}\left(\beta_{e} / \mu_{e}\right)}{k_{\perp}^{2}+\beta_{e} / \mu_{e}}\right)\right] \frac{1}{4 h}\left[\begin{array}{lllll}
1 & -5 & 3 & 1 & 0
\end{array}\right]
$$

for the diagonal blockmatrices $V_{l l}$ so that the diagonal elements of $V_{l l}$ increase like $k_{\perp}^{-2}$ for $k_{\perp} \rightarrow 0$. On the other hand, if we discretize $\nabla_{\|} \Phi$ via second-order centered differences, we get

$$
V_{l l}=\alpha_{e} v_{l}\left[\frac{1}{4 h}\left[\begin{array}{lllll}
1 & -5 & 3 & 1 & 0
\end{array}\right]+F_{0 l} \Delta v\left(\frac{1}{k_{\perp}^{2}}-\frac{2 v_{l} v_{i}\left(\beta_{e} / \mu_{e}\right)}{k_{\perp}^{2}+\beta_{e} / \mu_{e}}\right) \frac{1}{2 h}\left[\begin{array}{lll}
-1 & 0 & 1
\end{array}\right]\right]
$$

so that the diagonal elements of $V_{l l}$ are independet of $k_{\perp}$. The discretization of the $A_{\|}$term is not relevant for stability issues since the respective term in $V_{l l}$ is limited by $2 v_{\text {cut }}^{2}$ for $k_{\perp} \rightarrow 0$ as long as $\beta_{e} / \mu_{e} \neq 0$. (For $\beta_{e}=0$, the $A_{\|}$ term vanishes, anyway.) These considerations are qualitatively correct also for higher-order discretization schemes of either upwind or centered type. As will be shown in Section 4.2, although upwind methods according to cases (b) and (d) are stable, they are to be avoided on grounds of accuracy properties. In case (d), additional accuracy problems arise from finite differencing $g$ (which contains a contribution from $\left.A_{\|}\right)$and $A_{\|}$itself differently. In the remainder of this paper, we will therefore concentrate on centered methods. 


\subsection{Avoiding recurrence}

An important property of Eq. (8) may be investigated by neglecting the potentials $\phi$ and $A_{\|}$. It may then be written as a simple advection equation for $f(z, v, t)$ :

$$
\frac{\partial f}{\partial t}+v \frac{\partial f}{\partial z}=0
$$

A Fourier transformation of the $z$ coordinate leads to the equation

$$
\frac{\partial f_{k}}{\partial t}+i k v f_{k}=0
$$

for $f_{k}(v, t)$ which has the solution

$$
f_{k}(v, t)=f_{k}(v, t=0) e^{-i k v t}
$$

As is known from Van Kampen's theory of Landau damping [15], this system exhibits phase mixing, resulting in a decay of all moments of $f_{k}(v, t)$. In the course of this process, arbitrarily fine structures in velocity space are created. If Eq. (14) is solved numerically on an equidistant grid in velocity space with $v_{l}=l \Delta v$, the solution for a given $l$ can be written as

$$
f_{k}(t)=f_{k}(t=0) e^{-i k l \Delta v t}
$$

Note that for any $l$, the phase factor is equal to unity if $k \Delta v t=2 \pi$. This condition translates into the so-called recurrence time $t_{\mathrm{rec}}=(2 \pi) /(k \Delta v)$ after which the initial condition is restored. Since this is clearly unphysical, the finite resolution in velocity space effectively limits the total simulation time $T$. A typical initial value simulation of kinetic shear Alfvèn waves with $N_{v}=40$ is shown in Fig. 4. In this case, $t_{\mathrm{rec}}=69.1$.

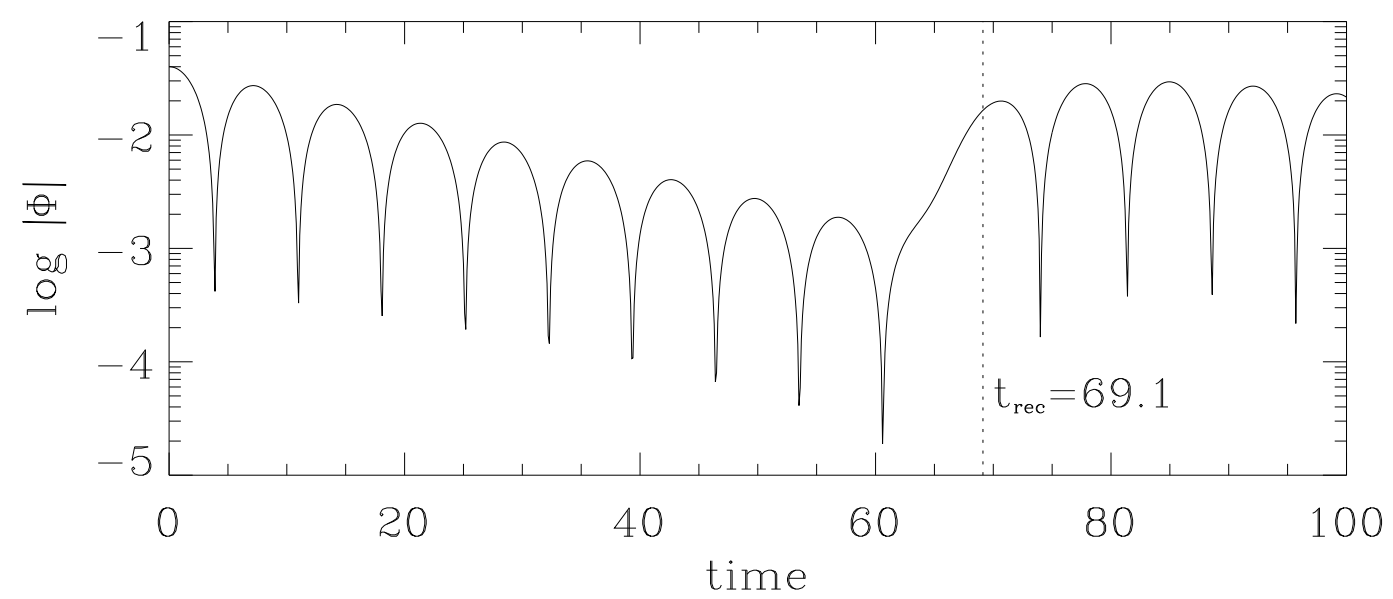

Fig. 4. Typical time trace of a simulation with $N_{v}=40$ : Around $t_{\mathrm{rec}}=69.1$, a recurrence phenomenon occurs. 
These considerations seem to indicate that $T$ can only be extended if $N_{v}$ is increased. However, there is an alternative solution to the recurrence problem. One can think of Eq. (16) as a wave in velocity space with wave number $k_{v}=k t$. Obviously, $k_{v}$ increases proportional to time. The Nyquist limit of the velocity grid, $k_{v, \text { Nyquist }} \Delta v=\pi$, is reached at $t=k_{v, \text { Nyquist }} / k=t_{\text {rec }} / 2$. Still finer scales are interpreted as $k_{v}=k t-k_{v \text {,Nyquist }}$, such that for $t=t_{\text {rec }}$ we have $k_{v}=0$. This suggests that recurrence can be avoided by stopping this cascade-like process in $k_{v}$ space before the Nyquist limit is reached. This can be achieved, for example, by means of a hypercollision operator like

$$
\frac{\partial f}{\partial t}=-\nu_{4} \frac{\partial^{4} f}{\partial v^{4}}
$$

Fourier transforming this equation to $k_{v}$-space, we obtain

$$
\frac{\partial f_{k_{v}}}{\partial t}=-\nu_{4} k_{v}^{4} f_{k_{v}}
$$

The solution of Eq. (19) is

$$
f_{k_{v}}(t)=f_{k_{v}}(t=0) e^{-\nu_{4} k_{v}^{4} t}
$$

Thus one can damp out fine scales in velocity space without affecting the larger scales.

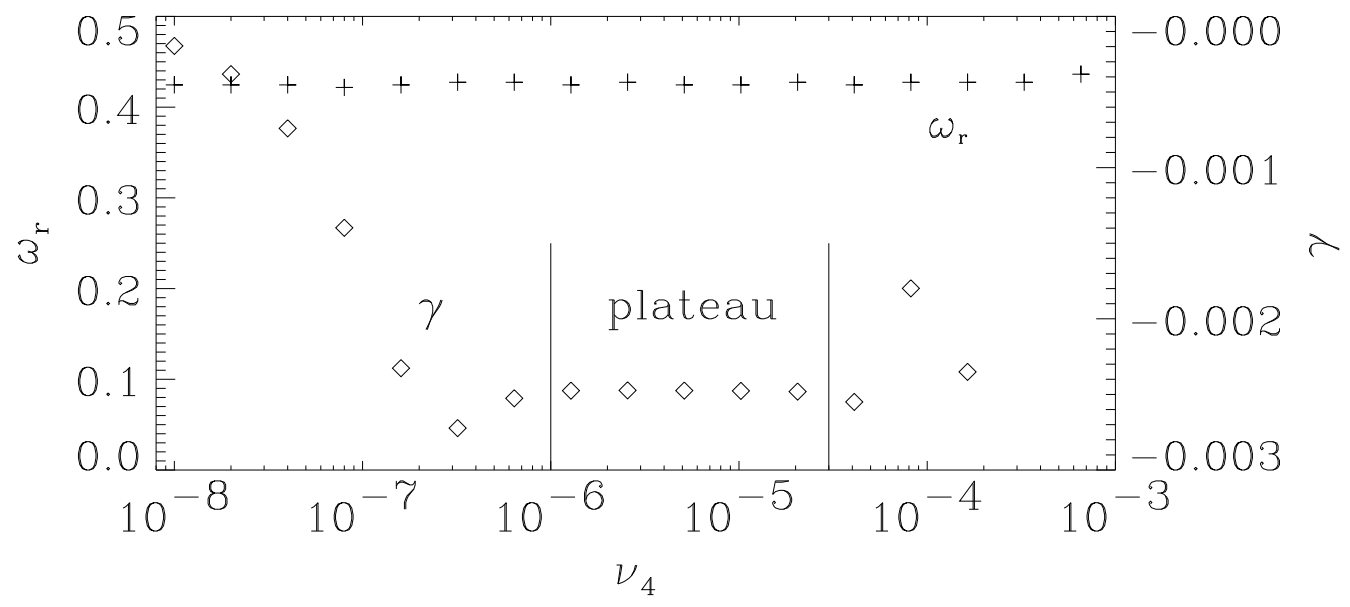

Fig. 5. Scan of the hypercollisionality parameter $\nu_{4}$ for $k_{\perp}=0.1, \beta_{e} / \mu_{e}=1$, $N_{v}=40$, and $N_{z}=32$.

The parameter $\nu_{4}$ which determines the damping strength must be chosen appropriately. If $\nu_{4}$ is too small, recurrence still occurs, if it is too big, the damping rate is controlled by hypercollisions, not by Landau damping. So we have to perform $\nu_{4}$ scans and search for a plateau in the damping rate curve. In Fig. 5, such a $\nu_{4}$ scan is shown for $k_{\perp}=0.1, \beta_{e} / \mu_{e}=1, N_{v}=40$, and $N_{z}=32$. There is a wide range of $\nu_{4}$ values over which the damping rate does not change substantially. Here like elsewhere in this paper, the damping rates 
are computed by means of a linear regression of $\log \phi_{k}(t)$, whereas the real frequencies are determined via the zeros of $\phi_{k}(t)$.

\section{Convergence tests}

\subsection{Velocity space resolution}

Having described the numerical methods used for solving the initial value problem, we now turn to convergence tests. First, we will address the issue of velocity space resolution. Given the fact that kinetic shear Alfvén waves are dissipative (kinetic) in nature, not reactive (fluid-like), one might think that the narrow Landau resonances in velocity space have to be strictly resolved in order to obtain correct results. This is not the case, however.

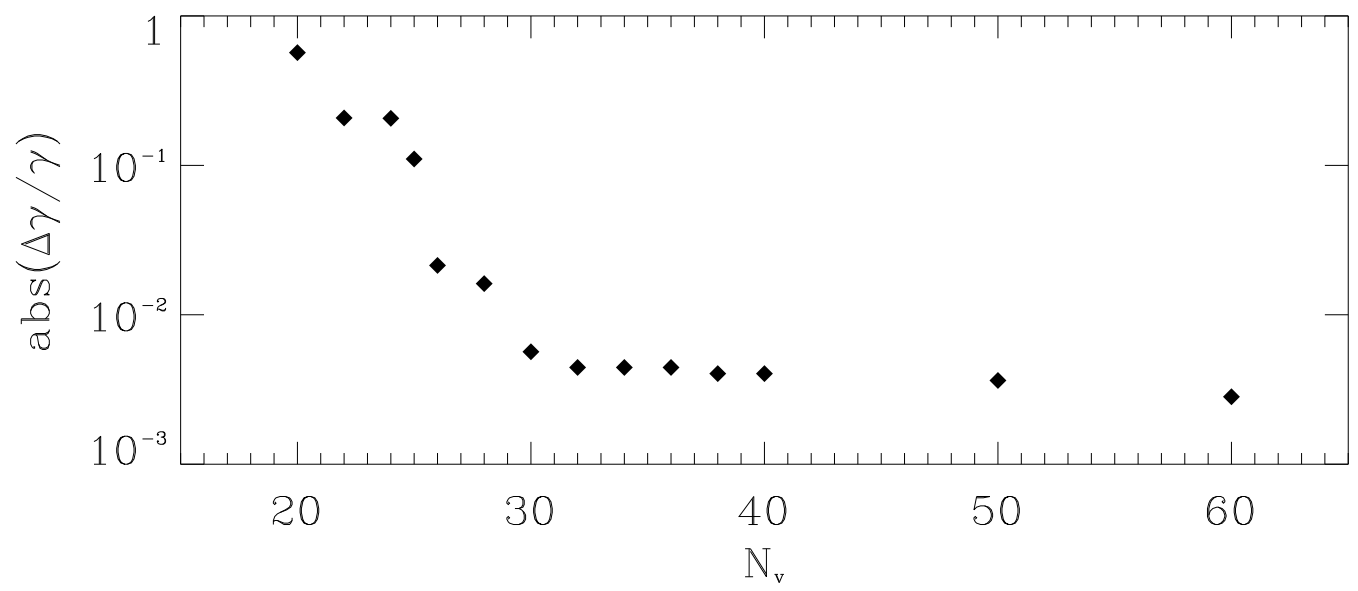

Fig. 6. Relative error in the damping rate, $|\Delta \gamma / \gamma|$, as a function of the number of points in velocity space, $N_{v}$.

The runs presented in this subsection all use the following parameters: $k_{\perp}=$ $0.1, \beta_{e} / \mu_{e}=1, N_{z}=32$, and $\nu_{4}=5 \cdot 10^{-6}$. Solving the dispersion relation directly, one obtains $\omega_{r}=0.42938$ and $\gamma=-0.002476$. These numbers are used to assess the quality of the initial value computations. In Fig. 6, the relative error in the damping rate is shown as a function of the number of points in velocity space, $N_{v} \cdot|\Delta \gamma / \gamma|$ drops in the range of less than about $1 \%$ for $N_{v} \gtrsim 25$. This result is somewhat surprising for a simple reason. Writing the resonance denominator as

$$
\frac{1}{\omega-\alpha_{e} v_{\|} k_{\|}}=\frac{\omega_{r}-\alpha_{e} v_{\|} k_{\|}}{\left(\omega_{r}-\alpha_{e} v_{\|} k_{\|}\right)^{2}+\gamma^{2}}-i \frac{\gamma}{\left(\omega_{r}-\alpha_{e} v_{\|} k_{\|}\right)^{2}+\gamma^{2}},
$$

one finds that its imaginary part has the form of a Lorentz curve with a fullwidth-at-half-maximum (FWHM) of $2|\gamma|$. This means that there is a Landau 
resonance in $v_{\|}$space around $\omega_{r} /\left(\alpha_{e} k_{\|}\right)$with a FWHM of $\delta v_{\|}=2|\gamma| /\left(\alpha_{e} k_{\|}\right)$. Using an equidistant grid with $\Delta v_{\|}=\left(2 v_{\text {cut }}\right) / N_{v}, \Delta v_{\|} \lesssim \delta v_{\|}$translates into $N_{v} \gtrsim 734$. In practice, we get away with about $1 / 30$ of that number, i.e., we do not need to resolve the fine-scale Landau resonances in velocity space. The structure of $f_{k} / \phi_{k}$ in $v_{\|}$space as it occurs in the simulation is shown in Fig. 7. For comparison, a theoretical prediction based on Eqs. (4) and (6) is also depicted.
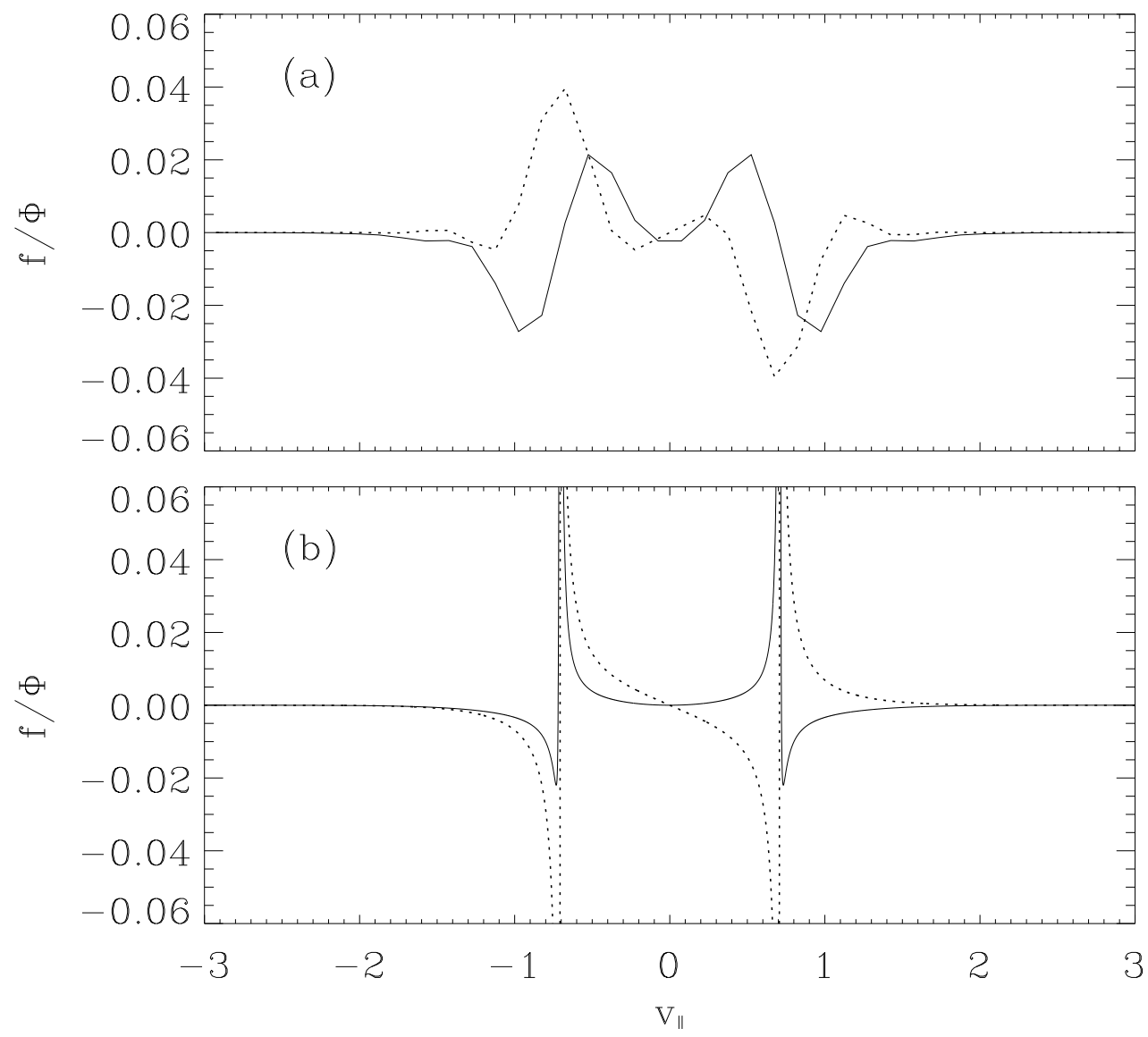

Fig. 7. Velocity space structure of $f_{k} / \phi_{k}$ from an initial value computation (a) and from theory (b). The real and imaginary parts are denoted, respectively, with solid and dotted lines. The Landau resonances are located around $\left|v_{\|}\right|=\omega_{r} /\left(\alpha_{e} k_{\|}\right) \approx 0.71$.

It must be emphasized that we have not attempted to minimize the number of velocity space points by use of non-equidistant grids and/or higher-order $v_{\|}$space integrators. One of the simplest generalizations would be to divide the domain $\left[-v_{\text {cut }}, v_{\text {cut }}\right]$ into two subdomains, a better resolved one located around the origin and encompassing the resonance region, and a less resolved one representing the tails.[10] But even for a simple equidistant grid, we find that the errors can be reduced if all grid points are shifted collectively by up to $\Delta v / 2$ such that the resonance region is better covered with grid points. 
Moreover, it should be kept in mind that the results presented in this subsection are strongly problem dependent. The required number of velocity space points may vary substantially with the type of wave or instability under consideration. Employing the gyrokinetic Vlasov code gene [14], we have found, for example, that toroidal ion temperature gradient instabilities are well represented by about $12 \times 6$ points in $\left(v_{\|}, \mu\right)$ space. Slab-type instabilities, on the other hand, may require more points in the $v_{\|}$direction (the number of points required in the $\mu$ direction tends to almost always stay small).[10] In general, one can say that reactive (fluid-like) modes are easier to resolve than dissipative (kinetic) modes.

\subsection{Configuration space resolution}

Next, the resolution requirements for the $z$ direction are investigated. Here we expect (and find) a dependence on the employed finite difference scheme. In Fig. 8, the results of initial value computations are compared with those of the dispersion relation for three different discretization methods: (a) fourth-order centered, (b) second-order centered, (c) second-order upwind for $f$ and secondorder centered for $\phi$. Obviously, the fourth-order centered scheme outperforms the other two, requiring less than 10 grid points in the spatial domain. The other parameters are $k_{\perp}=0.1, \beta_{e} / \mu_{e}=1, N_{v}=40$, and $\nu_{4}=5 \cdot 10^{-6}$. In this case, the dispersion relation yields $\omega_{r}=0.42938$ and $\gamma=-0.002476$.

The results of the initial value computations lie on straight lines in this log-log plot, reflecting the power law dependence of the relative error on $N_{z}$. Naturally, the slope depends on the discretization scheme. It is possible to predict the results of Fig. 8 analytically by inspection of a modified dispersion relation corresponding to the spatially discretized system. The latter has the form

$$
k_{\perp}^{2}+\left(\frac{Q_{\phi}}{Q_{f}}-\frac{2 \hat{\beta}}{\hat{\mu}} \bar{\omega}^{2}\right)(1+\bar{\omega} Z(\bar{\omega}))=0
$$

which depends on the finite difference scheme for the derivatives of $f$ and $\phi$ via the spectral functions $Q_{f}$ and $Q_{\phi}$. Moreover, $\bar{\omega}=\omega /\left(\alpha_{e} k_{\|}\right)$is changed to $\bar{\omega}=\omega /\left(\alpha_{e} k_{\|} Q_{f}\right)$. The relevant spectral functions are (using $\zeta=k_{\|} h$ ): 


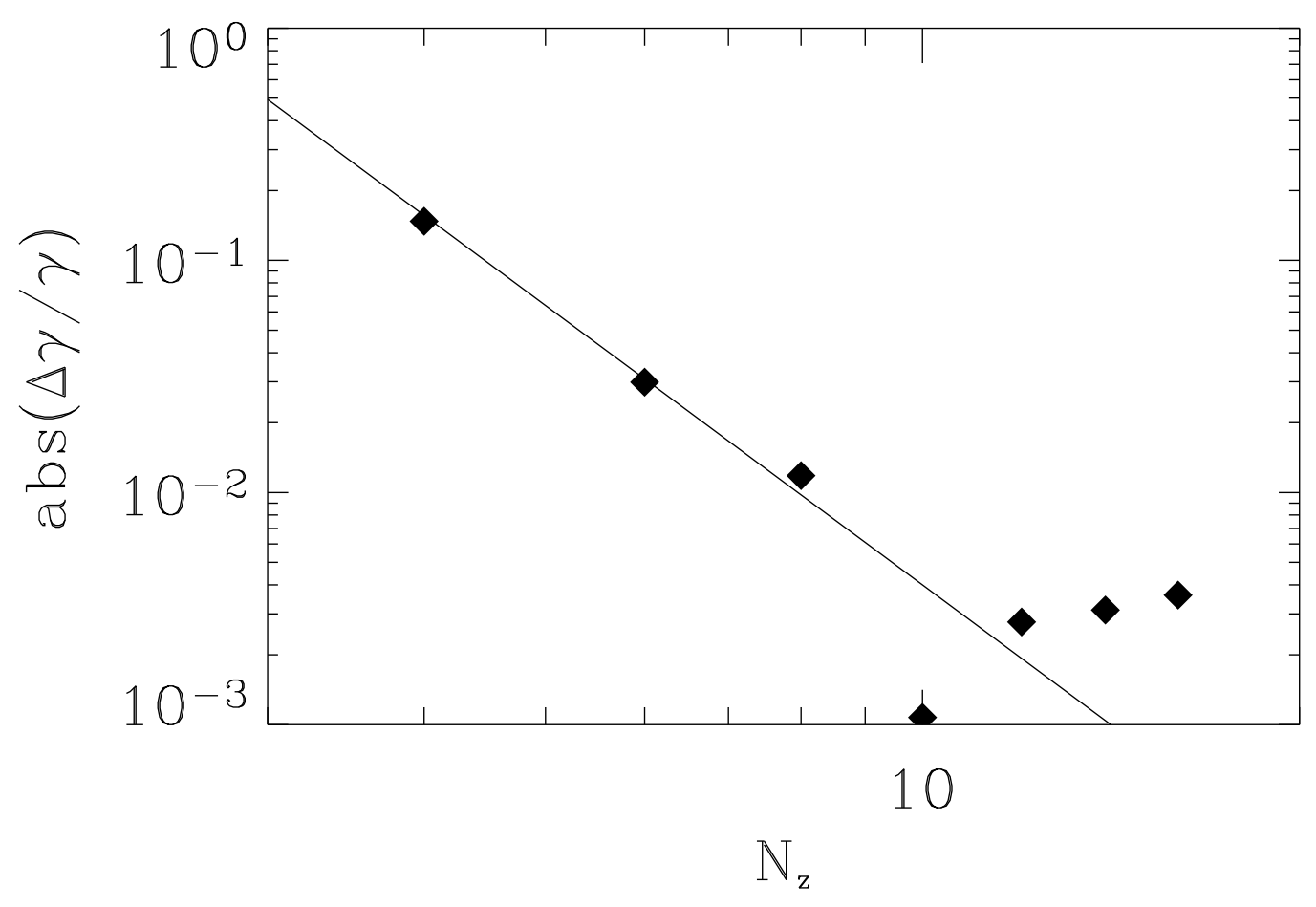

Fig. 8. Relative error in the damping rate, $|\Delta \gamma / \gamma|$, as a function of $N_{z}$ for three different discretization methods: (a) fourth-order centered, (b) second-order centered, (c) second-order upwind for $f$ and second-order centered for $\phi$. For comparison, analytic predictions are also shown (solid lines).

$$
\begin{aligned}
\text { second-order centered : } \quad Q(\zeta)= & \frac{\sin (\zeta)}{\zeta}, \\
\text { fourth-order centered : } \quad Q(\zeta)= & \frac{-\sin (2 \zeta)+8 \sin (\zeta)}{6 \zeta} \\
\text { second-order upwind : } \quad Q(\zeta)= & \frac{-\sin (2 \zeta)+6 \sin (\zeta)}{4 \zeta} \\
& +i \frac{-\cos (2 \zeta)+4 \cos (\zeta)-3}{4 \zeta}
\end{aligned}
$$

They are defined via $\nabla_{\|} \rightarrow i k_{\|} Q(\zeta)$.

If we use the same sort of finite differences for both $f$ and $\phi$, we have $Q_{\phi}=$ $Q_{f}=Q$. This means that the discretized dispersion relation differs from the continuous one only in the meaning of $\bar{\omega}$. The resulting complex frequencies just need to be rescaled by a factor of $Q$. We thus obtain $|\Delta \gamma / \gamma|=|Q-1|$. For second-order centered differences, Eq. (23) yields

$$
|\Delta \gamma / \gamma|=\zeta^{2} / 6+\mathcal{O}\left(\zeta^{4}\right)
$$


Analogously, for fourth-order centered differences one gets

$$
|\Delta \gamma / \gamma|=\zeta^{4} / 30+\mathcal{O}\left(\zeta^{6}\right)
$$

with the help of Eq. (24). These two functions are shown for comparison in Fig. 8. They agree quite nicely with the results from the initial value simulations.

Choosing a second-order upwind discretization for the distribution function $f$ together with a second-order centered scheme for the electrostatic potential $\phi$, the calculation is similar only if the same $Q$ is used irrespective of the sign of $v_{\|}$. (Otherwise, the plasma dispersion function $Z$ is not recovered.) By means of this approximation, one may solve Eq. (22) numerically to get the solid line corresponding to case (c) in Fig. 8. It reproduces the initial value results fairly well. However, the slope of this line is of the order of -3 and not -2 like expected for a second-order accurate scheme. This puzzle may be solved by further analyzing Eq. (22). Setting $Q_{\phi} / Q_{f}=1$, one obtains again a rescaled value of $\omega$, but this time the factor $Q_{f}$ is complex valued, $Q_{f}=Q_{1}+i Q_{2}$. The relative error turns out to be $|\Delta \gamma / \gamma| \approx\left|Q_{1}-1+\left(\omega_{r} / \gamma\right) Q_{2}\right|$ or

$$
|\Delta \gamma / \gamma| \approx \zeta^{2} / 12-\left(\omega_{r} / \gamma\right) \zeta^{3} / 8+\mathcal{O}\left(\zeta^{4}\right)
$$

Since $\omega_{r} / \gamma \approx-173.417$, the third-order term is dominant in the parameter range we are interested in. This explains the seemingly "wrong" slope of curve (c) in Fig. 8. Moreover, Eq. (28) reveals that any finite difference scheme for $\nabla_{\|}$whose spectral function $Q$ possesses a finite imaginary part, leads to large relative errors if $\left|\gamma / \omega_{r}\right| \ll 1$. This is the case for $k_{\perp} \ll 1$ and/or $\beta_{e} / \mu_{e} \gg 1$. Upwinding $g$ while center-differencing $\phi$ and $A_{\|}$makes matters even worse. This is because the contribution of $A_{\|}$to $g$ and $A_{\|}$itself (these two terms cancel out in an analytic formulation of the basic equations) leave a finite residuum when finite differenced differently. As far as the parallel dynamics of kinetic shear Alfvén waves are concerned, upwind schemes are therefore clearly inferior to centered schemes.

\section{$5 \quad$ Parameter scans}

Having tested the convergence properties of various numerical schemes, we are now in a position to do parameter scans. As was mentioned before, the two key quantities are the electron plasma beta (normalized to the electron-to-ion mass ratio), $\beta_{e} / \mu_{e}$, and the perpendicular wavenumber, $k_{\perp}$. But before we actually present the simulation results, we will derive some analytic results which may be used for comparison later. 


\subsection{Analytic results (limiting cases)}

The dispersion relation, Eq. (7), contains the plasma dispersion function $Z$ which, in general, must be evaluated numerically. Only in the limit of small or large arguments do analytic expressions exist.[11] For $|\bar{\omega}| \ll 1$, we have

$$
Z(\bar{\omega})=i \pi^{1 / 2} \exp \left[-\bar{\omega}^{2}\right]+\sum_{\nu} c_{\nu} \bar{\omega}^{\nu}, \quad \nu \in\{1,3,5, \ldots\}
$$

where the power series contains only odd powers of $\bar{\omega}$. Inserting this expression into Eq. (7), we see that it turns into a polynomial equation of infinite order. And this means that it possesses infinitely many solutions. A representative example of the positions of solutions in the complex $\bar{\omega}$ plane was shown in Fig. 1. We will be mainly interested in the one solution with the lowest damping rate since it dominates the long-time behavior of any initial-value problem (assuming that it is excited at all). Analytically, we may recover it by setting all coefficients $c_{\nu}$ to zero and replacing the exponential by unity. Eq. (7) then yields

$$
\bar{\omega}_{r}^{2}=\frac{1}{2} \frac{\mu_{e}}{\beta_{e}}\left(1+k_{\perp}^{2}\right), \quad \bar{\gamma}=-\frac{\pi^{1 / 2}}{4} \frac{\mu_{e}}{\beta_{e}} k_{\perp}^{2} .
$$

Here, we have evaluated the real and imaginary parts of Eq. (7) separately, at the same time assuming that $\left|\gamma / \omega_{r}\right| \ll 1$. A post-hoc self-consistency check shows that this assumption is only satisfied if $\beta_{e} / \mu_{e} \gg 1$ (and/or $k_{\perp} \ll 1$ ). In the opposite limit, $|\bar{\omega}| \gg 1$, an asymptotic expansion of $Z$ [11] yields (to lowest order)

$$
\bar{\omega}_{r}^{2}=\frac{\mu_{e}}{2} \frac{1}{\mu_{e} k_{\perp}^{2}+\beta_{e}} .
$$

This has important consequences for the numerical treatment of kinetic shear Alfvén wave in the appropriate limit. For $\beta_{e} \ll \mu_{e} k_{\perp}^{2}, \bar{\omega}_{r}$ scales like $k_{\perp}^{-1}$. In a simulation, the highest frequency is thus set by the smallest finite value of $k_{\perp}$. If a time-explicit numerical scheme is employed, increasing the perpendicular box size further increases $\bar{\omega}_{r}$ and in turn reduces the time step because of the numerical stability requirement $\omega_{r} \Delta t \lesssim 1$. For this reason it is often highly advisable (e.g., in gyrokinetic turbulence computations) to use finite values of $\beta_{e} / \mu_{e}$ to avoid these problems - even if the physics under consideration may be basically electrostatic in nature.

We note in passing that in unnormalized units, the real frequencies given in Eqs. (30) and (31) read, respectively,

$$
\omega_{r}^{2}=k_{\|}^{2} v_{A}^{2}\left(1+k_{\perp}^{2} \rho_{s}^{2}\right) \quad[\text { high } \beta \text { limit }]
$$


and

$$
\omega_{r}^{2}=\frac{k_{\|}^{2} v_{A}^{2}}{1+k_{\perp}^{2} \delta_{e}^{2}} \quad[\text { low } \beta \text { limit }] .
$$

Fluid models of kinetic shear Alfvén waves, on the other hand, lead to [16]

$$
\omega_{r}^{2}=k_{\|}^{2} v_{A}^{2}\left(\frac{1+k_{\perp}^{2} \rho_{s}^{2}}{1+k_{\perp}^{2} \delta_{e}^{2}}\right) .
$$

\subsection{Dependence on $\beta_{e} / \mu_{e}$ and $k_{\perp}$}

The following numerical results have been obtained using second-order centered differences for $\nabla_{\|}$together with a third-order ERK (Heun) time stepping algorithm. We have employed $N_{z}=32$ points in the spatial direction and $N_{v}=40$ points in velocity space. The hypercollisionality parameter $\nu_{4}$ was set to $5 \cdot 10^{-6}$. Only for cases in which $\gamma \lesssim 10^{-5}$, we used more (up to $N_{v}=140$ ) velocity space points, an extended velocity space domain (up to $v_{\text {cut }}=4$ ), and a reduced hypercollisionality parameter (of the order of $\nu_{4}=5 \cdot 10^{-7}$ ). For $k_{\perp}=0.3$, one obtains the results shown in Fig. 9. They agree well with the predictions from the dispersion relation over 4 orders of magnitude in $\beta_{e} / \mu_{e}$. In the $\beta_{e} / \mu_{e} \gg 1$ range, the predicted scalings [see Eq. (30)] of $\omega_{r} \propto\left(\beta_{e} / \mu_{e}\right)^{-1 / 2}$ and $\gamma \propto\left(\beta_{e} / \mu_{e}\right)^{-1}$ are recovered.

A $k_{\perp}$ scan at $\beta_{e} / \mu_{e}=1$ is shown in Fig. 10. As suggested by Eq. (30), $\omega_{r}$ is almost constant and $\gamma \propto k_{\perp}^{-2}$. The simulation results again compare very favorably with the solutions of the dispersion relation - even for values as low as $k_{\perp}=0.0025$ (corresponding to a perpendicular box size of more than $\left.2500 \rho_{s}\right)$. Note that in the limit $k_{\perp} \rightarrow 0$, kinetic shear Alfvén waves transition into ideal MHD Alfvén waves, characterized by $\omega_{r}^{2}=k_{\|}^{2} v_{A}^{2}$ and $\gamma=0$ in unnormalized units. This MHD limit is well captured by the present Vlasov scheme.

\subsection{Transition to electron sound waves at high $k_{\perp}$}

Replacing the driftkinetic approach [Eqs. (4) and (6)] by a gyrokinetic one [4], the graphs in Fig. 10 can be extended to smaller perpendicular scales, $k_{\perp} \gtrsim 1$. This is shown in Fig. 11, which has been produced by means of the gyrokinetic code gene.[14] In this case, the parallel gradients are again discretized via second-order centered differences, and a third-order ERK (Heun) time stepping scheme is used. Thus the numerics is basically the same as that described and studied in the present paper. As parameters we used $\beta_{e} / \mu_{e}=10$, $\hat{\epsilon}=18360, N_{z}=32, N_{v}=61$, and $N_{\mu}=8$ (gene employs two velocity space variables, the parallel velocity $v_{\|}$and the magnetic moment $\mu$ ). Here, instead 


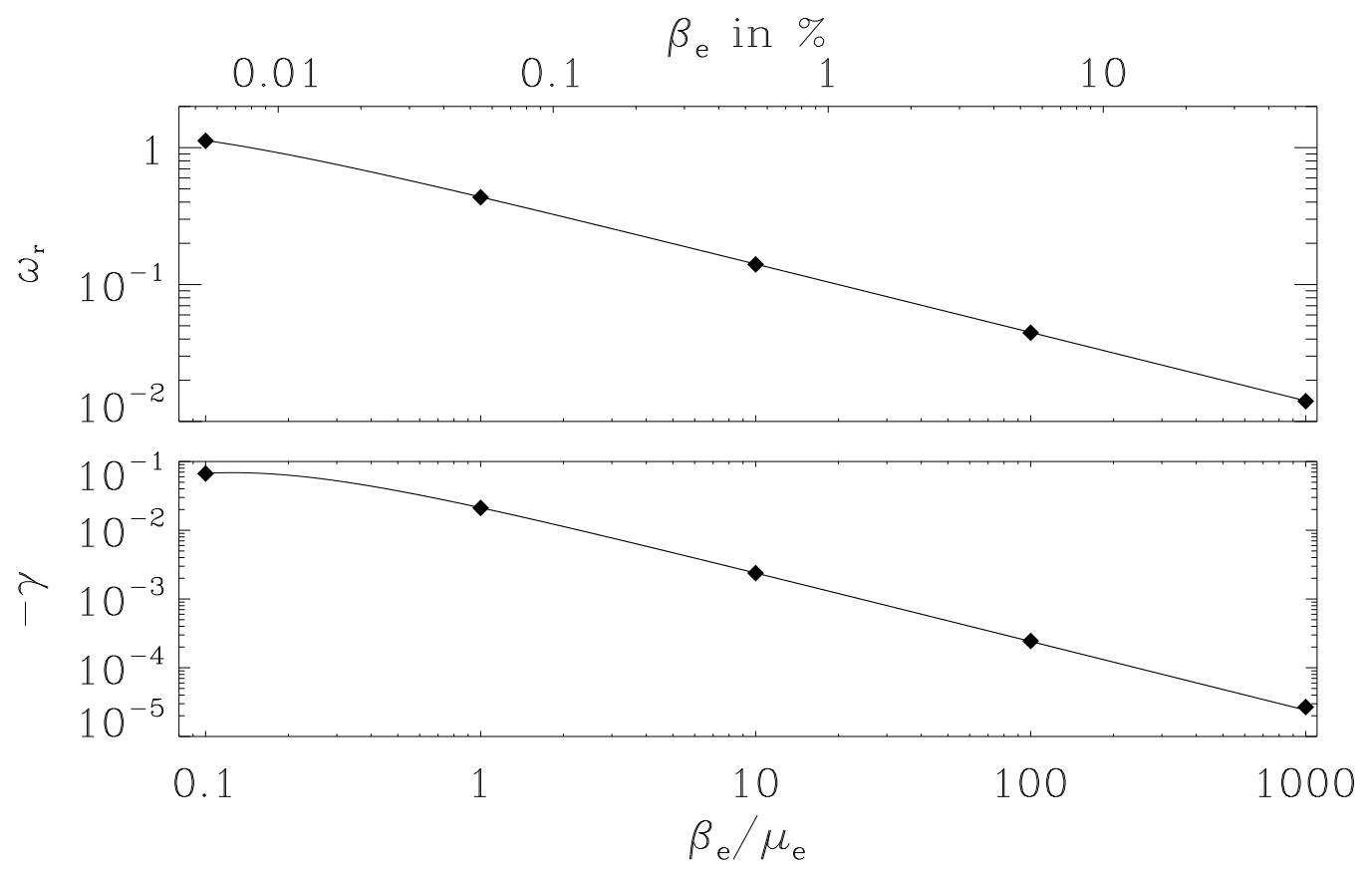

Fig. 9. Real frequencies and damping rates of kinetic shear Alfvén waves as a function of $\beta_{e} / \mu_{e}$ for $k_{\perp}=0.3$. The numerical solution of the corresponding dispersion relation is shown as a solid line for comparison.
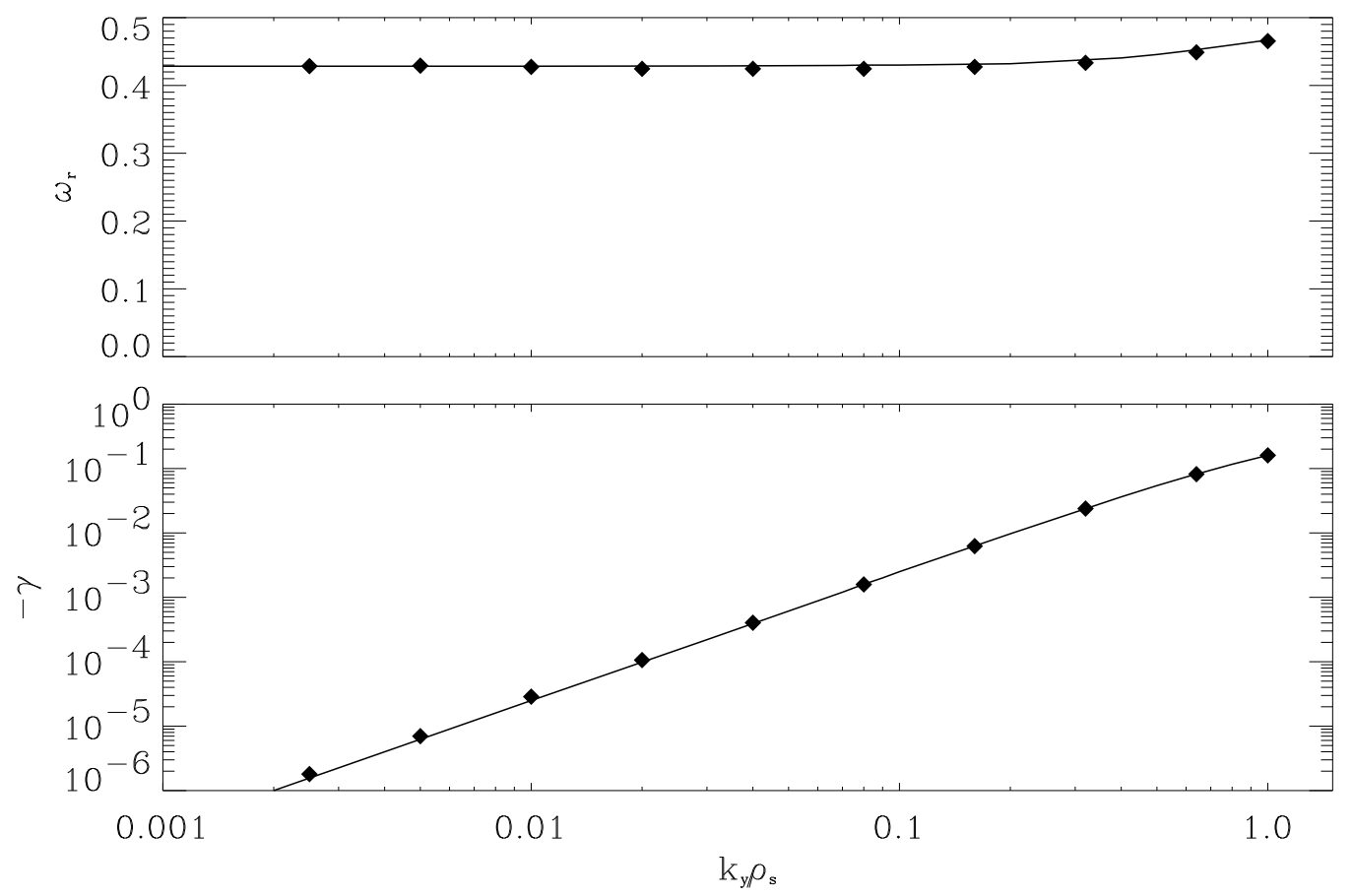

Fig. 10. Real frequencies and damping rates of kinetic shear Alfvén waves as a function of $k_{\perp}$ for $\beta_{e} / \mu_{e}=1$. The numerical solution of the corresponding dispersion relation is shown as a solid line for comparison. 
of using hypercollisions we simply increased $N_{v}$ to treat the weakly damped modes at low $k_{\perp}$, thus extending the recurrence time up to several 100 time units. The dispersion relation, Eq. (7), was also generalized to include finite Larmor radius effects. As can be seen in Fig. 11, the gene results and the solutions of the gyrokinetic dispersion relation agree quite well over a wide range of $k_{\perp}$. For $k_{\perp} \gg 1$, kinetic shear Alfvén waves become electron sound waves, the exact mirror image of ion sound waves. In particular, the ions are adiabatic at high $k_{\perp}$, leaving the dynamics entirely up to the electrons. For ion sound waves, driftkinetic theory yields

$$
\omega_{r}^{2}=k_{\|}^{2} \frac{\left(T_{e}+3 T_{i}\right) / m_{i}}{1+k_{\perp}^{2} \rho_{s}^{2}}
$$

in unnormalized units. Exchanging the species labels, setting $T_{e} / T_{i}=1$, and normalizing according to Table 1 , one obtains

$$
\bar{\omega}_{r}^{2}=\frac{2}{1+\mu_{e} k_{\perp}^{2}} .
$$

For $k_{\perp} \sim 15$, this formula yields $\omega_{r} \sim 0.6$, in good agreement with Fig. 11 . As expected, one finds that $\left|\gamma / \omega_{r}\right| \sim 1$, i.e., electron sound waves are strongly Landau damped. Finally, we would like to point out that the driftkinetic estimates for $\omega_{r}$ and $\gamma$ as given by Eq. (32) hold way beyond their strict range of validity, $k_{\perp} \ll 1$. As can be inferred from Fig. 11, the driftkinetic results give reasonable approximations up to $k_{\perp} \sim 1$.

\section{Summary}

We have examined kinetic shear Alfvén waves in homogeneous magnetoplasmas by means of Vlasov simulation. To this end, we solved the driftkinetic version of the Vlasov-Maxwell equations on a grid in $(1+1) \mathrm{D}$ phase space and compared the results to the numerical solutions of a respective dispersion relation. Very good agreement is obtained over a wide range of the two key parameters $\beta_{e} / \mu_{e}$ and $k_{\perp}$, even as $k_{\perp}^{2} \mu_{e} / \beta_{e} \rightarrow 0$. For an accurate reproduction of kinetic shear Alfvén waves, it turned out to be crucial to use a centered finite difference scheme for all spatial derivatives. If combined with an explicit Runge-Kutta time stepping algorithm, this requires the latter to be of third order or higher. Resolution tests revealed that a surprisingly low number of velocity space points is required, provided the recurrence problem for Landau damped modes on a phase space grid is dealt with (e.g. via hypercollisions). For long wavelengths, the ideal MHD results are recovered, whereas for short wavelengths, kinetic shear Alfvén waves transition to electron sound waves if the driftkinetic system is replaced by a gyrokinetic one. 

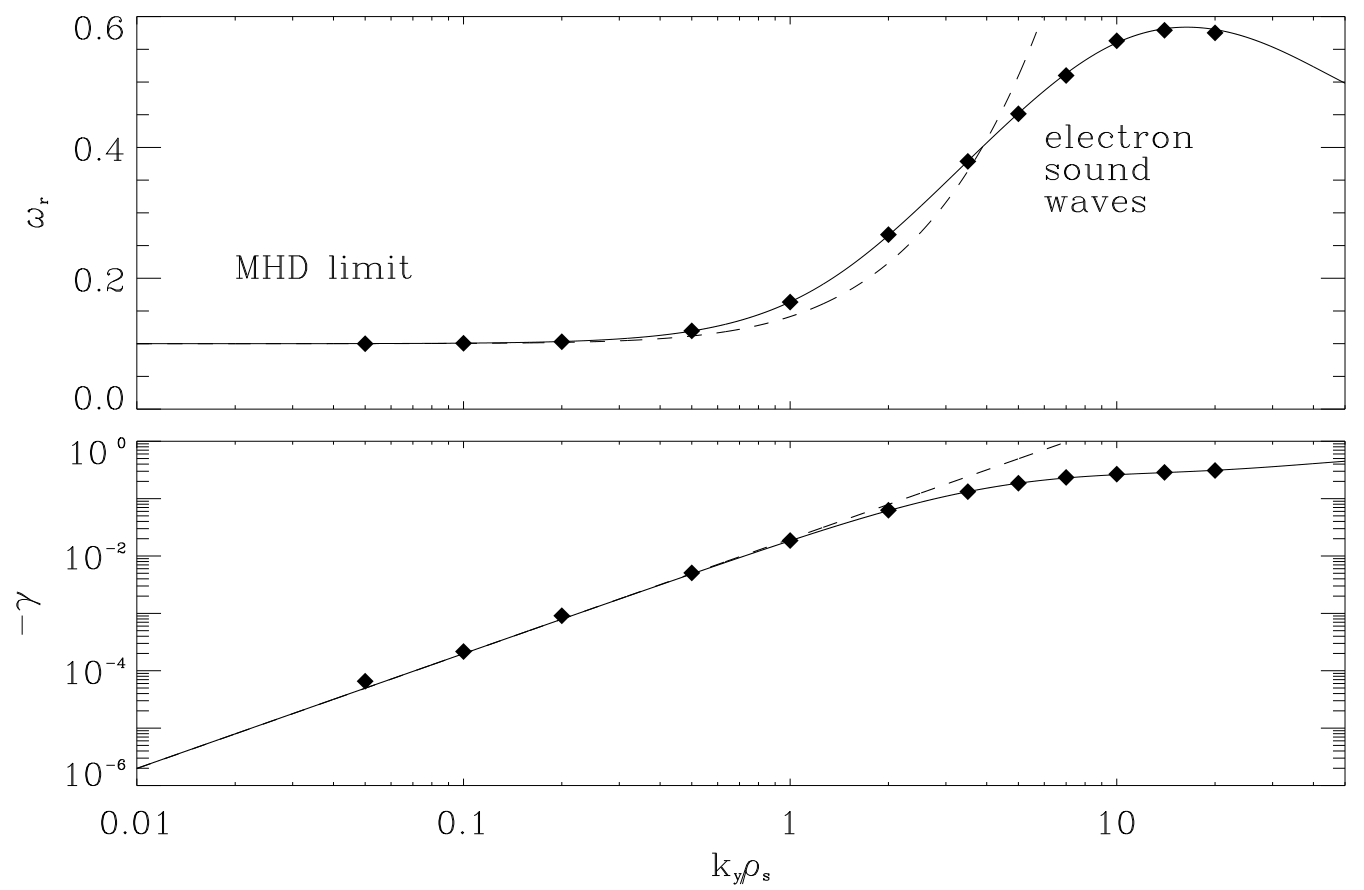

Fig. 11. Real frequencies and damping rates of kinetic shear Alfvén waves, computed with the gyrokinetic code gene. For $k_{\perp} \sim 10$, there is a transition to electron sound waves. The driftkinetic estimates are shown as dashed curves for comparison.

\section{Acknowledgement}

Frank Jenko would like to acknowledge helpful discussions with G. W. Hammett on the recurrence problem.

\section{References}

[1] A. B. Mikhailovskii, Instabilities in a Confined Plasma, IOP Publishing, Bristol, 1998.

[2] N. F. Cramer, The Physics of Alfvén Waves, Wiley, Berlin, 2001.

[3] R. D. Hazeltine and J. D. Meiss, Plasma Confinement, Addison-Wesley, Redwood City, CA, 1992.

[4] A. Brizard, J. Plasma Phys. 41, 541 (1989).

[5] F. Jenko and B. D. Scott, Phys. Plasmas 6, 2418 (1999).

[6] F. Jenko and B. D. Scott, Phys. Plasmas 6, 2705 (1999).

[7] C. K. Birdsall and A. B. Langdon, Plasma Physics via Computer Simulation, McGraw-Hill, New York, 1985. 
[8] F. Jenko and W. Dorland, Plasma Phys. Contr. Fusion 43, A141 (2001).

[9] T. P. Armstrong, R. C. Harding, G. Knorr, and D. Montgomery, in Methods of Computational Physics 9, edited by B. Alder, S. Fernbach, and M. Rotenberg (Academic, New York, 1970), p. 29

[10] F. Jenko, Comput. Phys. Commun. 125, 196 (2000).

[11] B. D. Fried and S. D. Conte, The Plasma Dispersion Function, Academic Press, New York, 1961.

[12] C. W. Nielson and H. R. Lewis, in Methods of Computational Physics 16, edited by B. Alder, S. Fernbach, and M. Rotenberg (Academic, New York, 1976), p. 367

[13] J. D. Lambert, Numerical Methods for Ordinary Differential Systems, Wiley, Chichester, 1991.

[14] F. Jenko, W. Dorland, M. Kotschenreuther, and B. N. Rogers, Phys. Plasmas 7, 1904 (2000).

[15] N. G. Van Kampen and B. U. Felderhof, Theoretical Methods in Plasma Physics, North-Holland, Amsterdam, 1967.

[16] B. Scott, Plasma Phys. Contr. Fusion 39, 1635 (1997). 